\title{
A New Science Gateway to Provide Decision Support on Carbon Capture and Storage Technologies
}

\author{
Yinzhi Wang \\ Indiana Geological \& Water Survey \\ Indiana University \\ Bloomington, IN 47405 \\ wangyinz@indiana.edu \\ Eroma Abeysinghe \\ Science Gateways Research Center \\ Pervasive Technology Institute, \\ Indiana University, \\ Bloomington, IN 47408 \\ eabeysin@iu.edu \\ Kevin Ellett \\ Indiana Geological \& Water Survey \\ Indiana University \\ Bloomington, IN 47405 \\ kmellett@indiana.edu
}

\author{
Sudhakar Pamidighantam \\ Science Gateways Research Center \\ Pervasive Technology Institute, \\ Indiana University, \\ Bloomington, IN 47408 \\ pamidigs@iu.edu \\ Suresh Marru \\ Science Gateways Research Center \\ Pervasive Technology Institute, \\ Indiana University, \\ Bloomington, IN 47408 \\ smarru@iu.edu \\ Marlon Pierce \\ Science Gateways Research Center \\ Pervasive Technology Institute, \\ Indiana University, \\ Bloomington, IN 47408 \\ marpierc@iu.edu
}

\author{
Sean Yaw \\ Los Alamos National Laboratory \\ Los Alamos, NM 87545 \\ yaw@lanl.gov \\ Marcus Christie \\ Science Gateways Research Center \\ Pervasive Technology Institute, \\ Indiana University, \\ Bloomington, IN 47408 \\ machrist@iu.edu \\ Richard Middleton \\ Los Alamos National Laboratory \\ Los Alamos, NM 87545 \\ rsm@lanl.gov
}

\begin{abstract}
Carbon dioxide capture and storage (CCS) is a promising technology for mitigating climate change, and its implementation is considered critical to meeting threshold targets for global warming in the 21 st century. We have developed a new science gateway application for the successful modeling software known as SimCCS that is used for evaluating complex, integrated CCS infrastructure. Using the Apache Airavata middleware and highperformance computing resources made available by the Extreme Science and Engineering Discovery Environment, we built the SimCCS Gateway to expand the tool's scalability for decision support and risk assessment. Case studies developed for evaluating a proposed CCS technology at Duke Energy's Gibson Station coalfired power plant in southwest Indiana demonstrate its improved ability in data analysis as well as risk assessment at various uncertainty levels. Further work is continuing to expand the functionality of both web and desktop clients, and to develop an active user group community in research and industry via the SimCCS Gateway interface.
\end{abstract}

Permission to make digital or hard copies of part or all of this work for personal or classroom use is granted without fee provided that copies are not made or distributed for profit or commercial advantage and that copies bear this notice and the full citation on the first page. Copyrights for third-party components of this work must be honored. For all other uses, contact the Owner/Author.
PEARC'18, July 22-26, 2018, Pittsburgh, PA, USA

(C) 2018 Copyright is held by the owner/author(s).

ACM ISBN 978-1-4503-6446-1/18/07.

https://doi.org/10.1145/3219104.3229244

\section{CCS CONCEPTS}

- Information systems $\rightarrow$ Decision support systems; • Information systems $\rightarrow$ Information systems applications

\section{KEYWORDS}

Carbon capture, Science gateways

ACM Reference format:

Y. Wang, S.Pamidighantam, S. Yaw, E. Abeysinghe, S. Marru, M. Christie, K. Ellett, M. Pierce, and R. Middleton. 2018. PEARC '18, Proceedings paper in Word Format, In Proceeding of Pearc'18 Conference, Pittsburgh, PA, USA, July 22-26, 2018, 3 Pages, https://doi.org/10.1145/3219104.3229244

\section{INTRODUCTION}

Deploying CCS technologies at industrial scale has been suggested to be a key approach for meeting the global warming threshold target of $2{ }^{\circ} \mathrm{C}$ or less. Successful CCS deployment requires consideration of the costs associated with capturing, storing, and 
transporting significant volumes of $\mathrm{CO}_{2}$. In addition, potential revenue from using the captured $\mathrm{CO}_{2}$ for enhanced oil recovery and other options is an important factor for industrial-scale projects to be economically viable. This requires careful techno-economic analysis of the integrated CCS system that includes not only $\mathrm{CO}_{2}$ sources and potential reservoir sinks, but also pipeline construction and the system performance over a multi-decadal time span. For industry to move forward with the large investments necessary to propel CCS technology deployment, it is critical to evaluate the complexities involved in the integrated system and to assess the risks associated with various uncertainties in the long-term performance of large-scale CCS projects.

A modeling tool known as SimCCS [1] has proven to be a promising approach for the integrated assessment of CCS technology proposals. Across the globe, researchers in government, academia, and industry have successfully implemented SimCCS to understand CCS infrastructure planning problems (e.g., [2] [3]). In this study, we extend SimCCS to address scalability and provide an adapted version available as software as a service through the SimCCS Science Gateway.

The SimCCS Gateway platform provides easy access to supercomputer resources within the Extreme Science and Engineering Discovery Environment (XSEDE), as well as local campus resources. It has three components: (1) a web client that provides basic access to data sets and supercomputers; (2) a desktop client for preparing and transferring models to and from supercomputers with corresponding solutions and visualization; and (3) Apache Airavata middleware [4][5] that communicates with supercomputer resources and the scripts and solvers necessary for running the models (Fig. 1). The open release of the SimCCS Gateway is designed to build a platform that enables other researchers to conduct CCS research at spatial resolutions and scales not previously attainable.

\section{DEVELOPING THE SIMCCS GATEWAY}

The critical infrastructure of the SimCCS Gateway is provided by the Apache Airavata science gateway framework. By default, the framework provides a PHP client for the web, but it also has APIs available for Java, Python, and $\mathrm{C}++$, allowing for alternative web and desktop programming capabilities. The web client hosted on the SciGaP server platform at Indiana University (IU) provides a simple interface for users to monitor and manage experiments on the gateway, and for administrators to securely manage users and computing and storage resources. In addition to local highperformance computing (HPC) resources on the IU Bloomington campus, our work aims to incorporate additional support within the XSEDE framework.

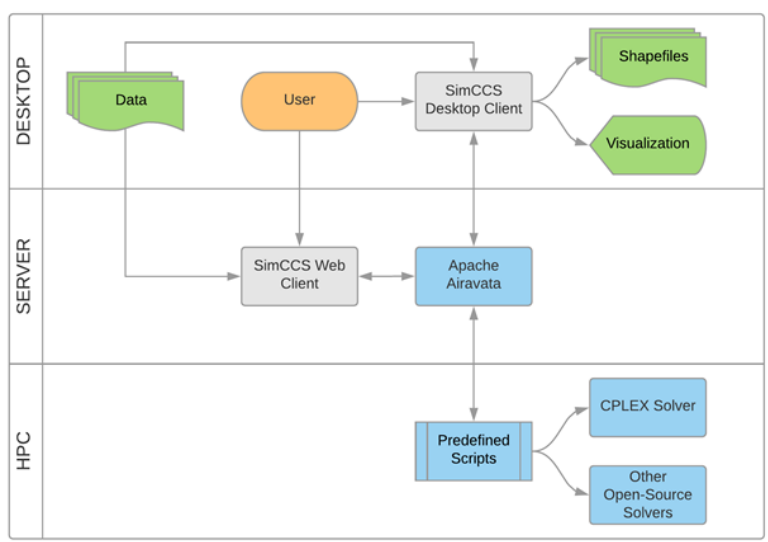

Figure 1. Flowchart of SimCCS Gateway workflow. The framework has three layers where users can interact with either the SimCCS Desktop Client or the SimCCS Web Client with all other complexities hidden. Both clients use Apache Airavata middleware to communicate with high-performance computing (HPC) resources.

The SimCCS Desktop Client Gateway is developed from a recently released open-source Java version of $\operatorname{SimCCS}^{2.0}$. The desktop version of the SimCCS gateway also works through Apache Airavata middleware to support the creation and management of computational experiments on remote resources, but it also has more sophisticated features than the web client. Its main functionalities include data input/output specification, along with pre-processing, visualization, analysis, and model formulation.

In addition to the basic functionality of visualizing all input data and solutions, we further improved the SimCCS Desktop Client from the original software by automatically generating weightedcost surfaces (necessary for pipeline network solutions) by combining the construction and right-of-way costs read from input files, eliminating the need for an additional bitmap file. Also, the improved geographic information system (GIS) built within the program not only allows the user to visualize the data and results, it also provides an interface for analyzing and manipulating data.

\section{CASE STUDIES AND EVALUATION}

To demonstrate the capabilities of the SimCCS Gateway, we performed a case study at Duke Energy's Gibson Station coal-fired power plant in southwestern Indiana. Because there are many mature oil and gas fields in this part of the Illinois Basin, CCS implementation can take advantage of ample opportunities for $\mathrm{CO}_{2}$ storage associated with $\mathrm{CO}_{2}$ injection for enhanced oil recovery. The first test scenario assumed a capture target of 15 megatons of $\mathrm{CO}_{2}$ per year $\left[\mathrm{Mt}\left(\mathrm{CO}_{2}\right) / \mathrm{yr}\right]$ over a proposed project length of 5 years, with $\mathrm{CO}_{2}$ storage targeted for oil and gas reservoirs at up to 42 discrete sink locations near the power plant. We demonstrated the improved data analysis ability of the SimCCS Gateway by conducting a two-step analysis that switches between a simplified candidate $\mathrm{CO}_{2}$ pipeline network for all 42 sinks and the complex shortest-path network for 12 selected sinks. Results demonstrated 
A New Science Gateway to Provide Decision Support on Carbon Capture and Storage Technologies

an improvement upon the prior network solution by achieving solution optimization. The associated reduction in the predicted financial cost of the project indicates that such analysis is critical for making well-informed business decisions. In the second test scenario, we illustrated how large ensemble model runs enabled by the SimCCS Gateway can be critical for risk assessment. We set a capture target of $15 \mathrm{Mt}\left(\mathrm{CO}_{2}\right) / \mathrm{yr}$ for 10 years with a variable injection cost rate that is normally distributed with different assumed standard deviations to simulate various levels of uncertainty. Aggregated, the solutions having 100, 1,000 and 10,000 iterations revealed how uncertainty is transferred into the final estimated project costs. The weak scaling of the simulation implies that one may easily scale up and solve even larger ensembles by increasing the number of CPUs (Table 1).

Another case study in the Ordos Basin, China ${ }_{2}$ is currently being undertaken as part of the bilateral collaborative effort known as the U.S.-China Clean Energy Research Center, Advanced Coal Technology Consortium. Workshops in both China and the United States are planned for the coming year to further integrate our academic and industrial partners into an international community of SimCCS Gateway developer and user groups.

Table 1. Solution time for solving problem ensembles using 64 cores.

\begin{tabular}{ll}
\hline Ensemble Size & Solution Time (s) \\
\hline 100 & 213 \\
1000 & 1208 \\
10000 & 11797 \\
\hline
\end{tabular}

\section{CONCLUSION}

A science gateway for evaluating $\mathrm{CO}_{2}$ capture and storage technologies is deployed using the Apache Airavata multi-tenanted gateway's middleware. The SimCCS Gateway release includes a desktop client that provides access to Airavata API functions and integrates SimCCS Java tools to load and visualize data, as well as to set up simulation experiments and results. Computational resources from IU and XSEDE are integrated into the gateway to enable solutions for the intensive network optimization process. Single and ensemble simulation capabilities implemented within the gateway provide optimal infrastructure network solutions based on the parameterization of the integrated CCS system components and uncertainty estimations. The gateway provides sharing and publishing capabilities, as well as a way to reproduce experiments using original data and modified input data for additional testing and assessment.

\section{FUTURE PLANS}

We are planning longer-term improvements including porting the solvers to a wide range of supercomputers within the XSEDE framework, improving the functionalities of the SimCCS web client, and reducing the dependency on strictly formatted source,
PEARC'18, Pittsburgh, Pennsylvania, July 2018

storage, and weighted-cost surface data. We also plan to extend the capability of the gateway with additional mapping and analysis tools on the web version, as well as adding pre-processing and federated integration with public GIS data for end-to-end processing of $\mathrm{CO}_{2}$ capture and storage. With consistent improvement and development over the course of the coming year, we are working toward our goal of providing the most powerful community software toolkit for evaluating CCS technologies.

\section{ACKNOWLEDGMENTS}

Primary funding for this project comes from the US-China Clean Energy Research Center, Advanced Coal Technology Consortium (award \#10-733-IU-2 from the West Virginia University Research Corporation). Additional support is provided by the Science Gateways Community Institute, NSF Award \#1547611 [6]. The SciGaP.org platform and Apache Airavata are supported by NSF Award \#1339774.

\section{REFERENCES}

[1] Middleton, R.S. and Bielicki, J.M., 2009. A scalable infrastructure model for carbon capture and storage: SimCCS. Energy Policy, 37(3), pp. 1052-1060.

[2] Middleton, R.S., Keating, G.N., Stauffer, P.H., Jordan, A.B., Viswanathan, H.S., Kang, Q.J., Carey, J.W., Mulkey, M.L., Sullivan, E.J., Chu, S.P., Esposito, R., Meckel, T.A., 2012. The cross-scale science of $\mathrm{CO}_{2}$ capture and storage: from pore scale to regional scale. Energy \& Environmental Science 5, $7328-7345$

[3] Keating, G.N., Middleton, R.S., Stauffer, P.H., Viswanathan, H.S., Letellier, B.C., Pasqualini, D., Pawar, R.J., Wolfsberg, A.V., 2011. Mesoscale Carbon Sequestration Site Screening and CCS Infrastructure Analysis. Environmental Science \& Technology 45, 215-222.

[4] Marru, S., Gunathilake, L., Herath, C., Tangchaisin, P., Pierce, M., Mattmann, C., Singh, R., Gunarathne, T., Chinthaka, E., Gardler, R. and Slominski, A., 2011, November. Apache Airavata: a framework for distributed applications and computational workflows. In Proceedings of the 2011 ACM workshop on Gateway computing environments (pp. 21-28). ACM.

[5] Pierce, M.E., Marru, S., Gunathilake, L., Wijeratne, D.K., Singh, R., Wimalasena, C., Ratnayaka, S. and Pamidighantam, S., 2015. Apache Airavata: design and directions of a science gateway framework. Concurrency and Computation: Practice and Experience, 27(16), pp. 4282-4291.

[6] Lawrence, K.A., Zentner, M., Wilkins-Diehr, N., Wernert, J.A., Pierce, M., Marru, S. and Michael, S., 2015. Science gateways today and tomorrow: positive perspectives of nearly 5000 members of the research community. Concurrency and Computation: Practice and Experience, 27(16), pp. 42524268. 